\title{
TOMA DE DECISIONES CENTRADAS EN LA FAMILIA EN LA ENFERMEDAD RENAL CRÓNICA AVANZADA. DE LA AUTONOMÍA INDIVIDUAL A LA AUTONOMÍA FAMILIAR O COMUNITARIA
}

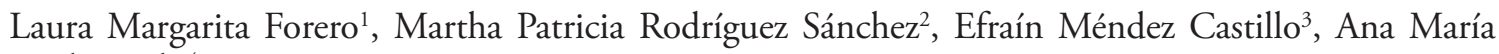
Medina Ch. ${ }^{4}$

\begin{abstract}
Resumen: El aumento de la tasa de incidencia y prevalencia de la enfermedad renal crónica plantea desafíos y dilemas éticos que tanto pacientes como familiares y profesionales de salud deben enfrentar diariamente. El objetivo de este artículo es presentar una reflexión sobre la manera como, para la toma decisiones, los pacientes recurren a terceros como partícipes, lo que requeriría un abordaje desde la autonomía solidaria. Se presenta el análisis de doce entrevistas semiestructuradas, realizadas a pacientes de 60 años o más, de distintos programas de la Unidad de Nefrología del Hospital Universitario San Ignacio, Colombia, entre 2017 2018. En este artículo se propone la necesidad de toma de decisiones centradas en la familia de los pacientes con enfermedades crónicas, antes de que su estado sea avanzado y los estados de inconsciencia impidan su autodeterminación, considerando la determinación social de la autonomía.
\end{abstract}

Palabras clave: enfermedad renal crónica, autonomía personal, toma de decisiones, ética, investigación cualitativa

Family Centered Decision Making in Advanced Chronic Kidney Disease. From individual autonomy to family or community autonomy

\begin{abstract}
The increase in the incidence and prevalence rate of Chronic Kidney Disease poses challenges and ethical dilemmas that patients, family members and health professionals must face daily. The objective of this article is to present a reflection on the way in which patients make recourse to third parties as participants in their decision-making, which would require an approach based on solidarity autonomy. The analysis of twelve semi-structured interviews conducted with patients 60 years of age and older, from different programs of the Nephrology Unit of the Hospital Universitario San Ignacio in the period $2017-$ 2018 Colombia, is presented. This article proposes the need for family-centered decision making of patients with chronic diseases, before their state is advanced and states of unconsciousness prevent their self-determination, considering the social determination of autonomy.
\end{abstract}

Key words: renal insufficiency chronic, personal autonomy, decision making, ethics, clinic, qualitative research

\section{Tomada de decisóes centradas na família na enfermidade renal crônica avançada. Da autonomia individual à} autonomia familiar ou comunitária

\begin{abstract}
Resumo: $\mathrm{O}$ aumento da taxa de incidência e prevalência da enfermidade renal crônica coloca desafios e dilemas éticos que tanto pacientes como familiares e professionais da saúde devem enfrentar diariamente. O objetivo deste artigo é refletir acerca de que, para a tomada de decisóes, os pacientes recorrem a terceiros como partícipes, o que a nosso juízo requer uma abordagem a partir da autonomia solidária. Se apresenta a análise de doze entrevistas semiestruturadas, realizadas com pacientes de 60 anos ou mais, de distintos programas da Unidade de Nefrologia do Hospital Universitário San Ignacio, Colômbia, entre 2017-2018. O artigo propóe a necessidade de tomada de decisões centradas na família dos pacientes com enfermidades crônicas, antes que seu estado esteja avançado e estados de inconsciência impeçam sua autodeterminaçáo, considerando a determinação social da autonomia.
\end{abstract}

Palavras chave: enfermidade renal crônica, autonomia pessoal, tomada de decisões, ética, investigação qualitativa

\footnotetext{
${ }^{1}$ Hospital Universitario San Ignacio, Programa de Postgrado, Pontificia Universidad Javeriana, Bogotá, Colombia. Correspondencia: la-forero@javeriana.edu.co

${ }^{2}$ Hospital Universitario San Ignacio, Pontificia Universidad Javeriana, Bogotá, Colombia

Correspondencia: m-rodriguezs@javeriana.edu.co

${ }^{3}$ Instituto de Bioética y Director programa Maestría en Bioética, Pontificia Universidad Javeriana. Bogotá Colombia Correspondencia: e.mendez@javeriana.edu.co

${ }^{4}$ Instituto de Envejecimiento, Facultad de Medicina, Pontificia Universidad Javeriana, Bogotá, Colombia

Correspondencia: medina.ana@javeriana.edu.co
} 


\section{Introducción}

La enfermedad renal crónica (ERC) es un problema de salud en constante crecimiento y de muy alto costo, que ocasiona un impacto negativo en la disponibilidad de los recursos para la atención de la población $(1,2)$. El proceso de toma de decisiones en los pacientes con enfermedad renal crónica avanzada (ERCA) es complejo y se debe tener en consideración tanto su edad como el estadio de su enfermedad, y la alta carga de comorbilidad y discapacidad asociada. No todos los pacientes son candidatos a las terapias de sustitución renal o, en algunas ocasiones, se deberá plantear la suspensión de estas, basados en el contexto clínico o en el momento en que la intervención se considere un acto fútil $(3,4)$. Estas características hicieron que la Sociedad Americana de Nefrología recomendara la planificación de decisiones anticipadas como la piedra angular en el manejo de los pacientes con $\operatorname{ERCA}(1,5)$. Adicionalmente la autonomía en los pacientes mayores, con enfermedades crónicas y progresivas puede verse mediada por la regresión psicomotriz, caracterizada por un retroceso emocional y físico de los estándares de independencia y autodeterminación de la persona(6).

Comúnmente, el derecho a morir dignamente (DMD) y las decisiones anticipadas al final de vida se espera sean tomadas en el marco de la relación médico-paciente como decisiones compartidas(7) e informadas, guiados las más de las veces por el principio de la autonomía, entendido como el respeto a la decisión del paciente como individuo, teniendo en consideración sus preferencias, es decir una atención centrada en la persona en una sociedad comprometida con las libertades individuales(8). Sin embargo, en la última década, la literatura científica ha comenzado a volcar su atención en el papel de la familia en la toma de decisiones(9), en la medida en que se reconoce que las decisiones son procesos complejos, no necesariamente individuales, que se toman de manera vincular con otros actores, incluyendo el médico tratante, la familia, la pareja, entre otros. Hay poca investigación acerca de las representaciones respecto de las voluntades anticipadas y el derecho a la muerte digna en esta población(10).

Teniendo en cuenta estos antecedentes, surgió la necesidad de realizar un estudio, de corte inter- pretativo, que permitiera "comprender las comprensiones, saber sobre los saberes y entender los entendimientos"(11) del derecho a morir dignamente y las voluntades anticipadas en esta población, motivo por el cual se realizó este trabajo de investigación usando la "teoría de las representaciones sociales" como herramienta teóricometodológica(12). En este artículo se analiza la actitud de pacientes a las decisiones anticipadas, y la subrogación de las decisiones a la familia en pacientes con ERCA(13).

El derecho a morir dignamente DMD se refiere al derecho de disponer de la propia vida. Cobija el hecho de morir sin dolor, con serenidad, acompañado y reconciliado consigo mismo y con los demás(14). Una de las herramientas de utilidad para hacerlo real son las voluntades anticipadas, que en Colombia se definen(15) como el instrumento que le permite a un individuo participar en la toma de decisiones relacionadas consigo mismo y con el cuidado o atención de su salud; en especial en el caso de incapacidad, limitación o para manifestar sus preferencias al final de la vida, si llegara el caso de que no pudiera expresar su voluntad o estuviera limitado para razonar. Existen mecanismos en los cuales la responsabilidad de la decisión recae sobre otros agentes, en este caso, la familia(16). El marco ético de actuación de un sustituto se rige por criterios subjetivos: de juicio sustitutivo o de mayor beneficio. Los criterios subjetivos incluyen la decisión previa del paciente, cuando es capaz, o mediante directrices directas anticipadas, o indirectas: poderes de representación sobre cómo ser tratado en momentos determinados. El criterio de juicio substitutivo implica que un sustituto elige lo que el paciente elegiría si fuese competente y estuviese a la par tanto de las opciones médicas, como de su situación clínica real, incluyendo la posibilidad de ser considerado incompetente. El criterio del mayor beneficio o mejor interés se refiere a que el sustituto elije lo que, en su juicio, mejor promueve los intereses del paciente y aquello que le proporciona el mayor bien(17). El sustituto, además de conocer bien al paciente, debe haber mantenido con él una relación íntima y saber cuáles fueron los valores y creencias a partir de los cuales configuró su proyecto de vida, puesto que el criterio del juicio sustitutivo, al igual que sucede con el criterio de la autonomía pura, se pre- 
senta como un modelo de decisión que pretende fundamentalmente ser respetuoso con el principio de autonomía del paciente(16).

La autonomía es una expresión polisémica que puede tener distintos alcances semánticos y conceptuales. En bioética se han desarrollado diferentes teorías al respecto, como señalan Beauchamps y Childress en el texto canónico de la bioética clínica. La teoría más consistente, y posiblemente la que fundamentó todas las otras, es la que se desprende de la noción kantiana de autonomía y consiste en permitir a cada individuo ser sí mismo, y eso es algo más amplio que tomar decisiones libremente. Esta noción kantiana de autonomía concibe al individuo y su voluntad como intrínseca, con voluntad para autodeterminarse. En esta acepción, el sujeto decide por sí mismo sin ninguna consideración que le sea impuesta externamente, sigue un deseo que le es propio. Esta voluntad para autodeterminarse es lo que lo constituye libre y a su vez responsable de sus acciones(18). En bioética clínica significa tener al paciente como "un fin en sí mismo", nunca como un medio, independiente de su edad, condición neurológica o psiquiátrica, pues abarca todas las condiciones de posibilidad de existir. Iosa, citando a J. S. Miller, enuncia que, además de no interferir en el curso de vida, el deber moral es promoverla activamente, lo que se traduce en el deber del médico por actuar a favor de la realización del plan de vida que cada uno tiene(19).

Para el caso de los pacientes aquí entrevistados, se identificó una tendencia a no decidir anticipadamente, sino a ceder la decisión a sustitutos. En este artículo se considera importante considerar las diferentes formas de entender el principio ético de respeto a la autonomía del individuo, en la medida en que devienen del problema de la misma noción de "individuo", pues de ella se desprende su estatus moral y, en consecuencia, el respeto debido a su historia y autodeterminación.

Algunos autores señalan, dentro de la corriente kantiana, la necesidad de distinguir entre los deseos de primer y segundo orden, siendo los primeros aquellos que emergen de manera espontánea, sin reflexión, y los segundos el fruto de un proceso de reflexión, juicio o posicionamiento del mismo individuo sobre los deseos de primer orden(19).
Esta forma de comprender la autonomía puede ser puesta en duda - como lo han hecho otros autores - señalando que esta libertad y autodeterminación solamente puede consolidarse como resultado de procesos sociales de los cuales hace parte el individuo, es decir que el individuo es un sujeto social per se, y que, por lo tanto, la autonomía no puede comprenderse como separada del contexto sociohistórico dentro del cual se desarrolla la persona. Las concepciones individualistas señalan que, en la medida en que la persona esté sujeta a la voluntad de otro, entonces será heterónoma más que autónoma respecto de las decisiones. Ramírez cuestiona las aproximaciones individualistas en la medida que exacerban el narcicismo, e invita, como contrapeso, a la integración de lo cognitivo, lo afectivo y lo social en el desarrollo de lo moral, considerando este paso indispensable para el desarrollo de la autonomía(20).

En un primer momento, la bioética clínica consideró la autonomía del paciente como un asunto puramente individual, en que cada paciente debía tomar decisiones de manera puramente aislada, y que lo conveniente era respetar ese ámbito privado y subjetivo. Con el tiempo se puso en evidencia que la experiencia individual de enfermedad hace parte de una red de relaciones e interdependencias, con lo cual las decisiones ético-clínicas que se deriven del ejercicio de su autonomía forman parte de una red de representaciones y valoraciones sociales que, necesariamente, van a afectar de forma positiva o negativa a sus interdependientes. Aquí emerge el concepto de "autonomía relacional". Así, para efectos de evaluar la toma de decisiones, el paciente debe ser entendido como un nodo de una red social compleja y heterogénea $(21,22)$.

Mientras la aproximación individualista de la autonomía plantea que la respuesta a qué hacer frente a emociones, sentimientos o deseos emergentes, es el juicio racional ${ }^{23}$, en el concepto de "autonomía solidaria" o "relacional" esta se construye en términos intersubjetivos, lo que conlleva la preocupación por el otro a nivel empático(20). De hecho, los estudios en neurobiología cuestionan cada vez más el concepto mismo de "juicio racional" en la toma de decisiones(23), ya que se está dilucidando la inmensa influencia de la esfera emocional, radicada en el área límbica y activamente conectada en el córtex prefrontal, en la toma de decisiones. 
Desde los estudios neurofisiológicos de Wilder Penfield en la década de 1950-1960 y los efectuados durante la segunda mitad del siglo XX, con estimulación de áreas cerebrales con electrodos, se consolidó la idea topográfica de que las decisiones racionales resultaban de procesos cognitivos superiores, que ocurrían exclusivamente en áreas del neocortex cerebral aislados de otras influencias. Se creía en una especialización de las funciones cerebrales por áreas topográficamente diferenciadas; por otra parte, los estudios sugerían que las emociones se radicaban exclusivamente en áreas específicas, como era la amígdala y en general el mesencéfalo. Se hablaba de dos cerebros: el racional y el emocional. No obstante, la mejor comprensión de las conexiones neurológicas, gracias a nuevas herramientas de exploración, empezó a revelar, a finales del siglo XX -1995 en adelante- que tal independencia y el vínculo causal unilineal no eran ciertos, sino, por el contrario, existía una intensa interconexión entre áreas cerebrales que se consideraban autónomas y especializadas. Así se puso en evidencia cómo las distintas y dispersas regiones cerebrales involucradas en emociones están implicadas en la cognición y viceversa. La memoria almacenada en lugares distintos también resultó ser determinante en la configuración de las decisiones. Hoy resulta absurdo pensar en las decisiones como fenómenos de pura racionalidad. Gracias a los estudios de Antonio Damasio y muchos otros, se ha demostrado, desde 2000 en adelante, la relevancia de las emociones en la toma de decisiones(24). Otra perspectiva sobre la "irracionalidad de las decisiones morales" puede ser encontrada a lo largo del texto Gobierno de las emociones, de Victoria Camps, en el que la autora busca distanciarse del racionalismo hegemónico y considera la complejidad de la toma de decisiones como uno de los problemas fundamentales de la ética contemporánea(25).

Proponemos entonces que los aspectos cognitivos y afectivos se articulan en la toma de decisiones, y en la autonomía que resulta de un ejercicio de equilibrio constante entre el contexto y las relaciones familiares y sociales. La autonomía solidaria plantea que esta solo se puede lograr en interacción, en el encuentro con otro que se considera como igual; y que exige la puesta en común de puntos de vista distintos. Vista así, la autonomía es solo una posibilidad en el desarrollo del ser humano, una potencialidad que requiere un proceso y continuidad en el tiempo, ya que, como ser social, es necesario el momento y espacio de diálogo para que, a partir del mejor interés solidario y por las personas involucradas, se tomen decisiones basadas en las creencias y valores del individuo, en el marco de formas de vida comunitarias que son su fuente(20).

\section{Metodología}

Este estudio corresponde a una investigación cualitativa-interpretativa. Su protocolo fue aprobado por Comité de Ética correspondiente a las instituciones involucradas, y teniendo como referencia la Declaración de Helsinki. De acuerdo con la legislación vigente en Colombia, corresponde a un estudio de riesgo mínimo. La recolección de información se efectuó de febrero de 2018 a febrero de 2019. Se realizaron doce entrevistas semiestructuradas, realizadas a pacientes de 60 años o más, tratados en la Unidad de Nefrología del Hospital Universitario San Ignacio, Bogotá, Colombia. El muestreo se realizó por tipificación, tomando como referencia la clasificación propuesta por la Kidney Disease: Outcome Quality Initiative K/ DOQI, que define la enfermedad renal crónica avanzada ERCA como aquella que cursa con un descenso grave del filtrado glomerular FG $<30$ $\mathrm{mL} / \mathrm{min} / 1,73 \mathrm{~m} 2(13)$. En el estudio se incluyó a pacientes del programa de prediálisis (PD), diálisis peritoneal (DP), hemodiálisis (HEMO) y trasplante renal (TR) (ver tabla 1). Se eligió a tres participantes por cada tipo de tratamiento de ERC, buscando dar cuenta de la experiencia de pacientes en las diversas etapas de la enfermedad. Los pacientes fueron contactados y, una vez expresaron su interés en participar, se les explicó el proyecto y los alcances de este, y se firmó el consentimiento informado. Un paciente se negó a participar de la entrevista. En todo momento se mantuvo la confidencialidad de la información. Para la realización de las entrevistas los pacientes podían estar acompañados de un familiar o cuidador, si así lo deseaban. La guía de entrevista se estructuró acorde a la teoría de las representaciones sociales, considerando tres componentes de estas: el campo de representación, la información y la actitud en torno a los derechos de los pacientes, el DMD y las voluntades anticipadas. Las entrevistas 
fueron realizadas por una residente de geriatría, miembro del equipo de investigación, quién también las transcribió, y quien no tenía injerencia directa sobre el curso del tratamiento, pero si una relación previa de confianza con los pacientes, establecida durante su rotación por el servicio. Adicionalmente se llevaban notas de campo durante las entrevistas y visitas al servicio. Una de las investigadoras principales es médica especialista de la Unidad, y fue quien contactó inicialmente a los pacientes. No realizó ella las entrevistas, con el fin de reducir el efecto de la relación médico-paciente en las respuestas. Los otros dos investigadores no tenían relación directa con el servicio o los pacientes. El análisis de las entrevistas transcritas se hizo de manera paralela por el resto del equipo interdisciplinario, identificando los contenidos pertinentes a cada componente de las representaciones sociales e identificando categorías emergentes asociadas a la toma de decisiones y autonomía, así como las barreras que se podían identificar en las entrevistas a los pacientes. Estos resultados son los que se presentan en este artículo.

\section{Resultados: la autonomía en los pacientes de ERCA mayores de 60 años}

Los pacientes oscilaron entre los 61 y 81 años (ver tabla 1). El tiempo de terapias de reemplazo renal era variado: los pacientes que se encontraban en hemodiálisis entre 2 y 10 años, diálisis peritoneal menos de un año y 2 ańos, trasplante renal entre 2 y 19 años, y prediálisis menos de 1 año y 2 ańos.

La condición de los pacientes y los tratamientos asociados los sujeta a largos tratamientos y visitas continuas a los hospitales. En el análisis de las entrevistas se identificó el género, las relaciones familiares y la religión como centrales en la toma de decisiones, y en directa relación con la autonomía frente a las decisiones de vida como durante las distintas fases de los tratamientos.

\section{La familia y las decisiones}

Del total de pacientes entrevistados, la mitad realizó las entrevistas con un acompañante familiar. Los pacientes que tenían documentos ya firmados contaban con un hijo o hija para la toma de decisiones, dejando todas las decisiones a criterio de sus hijos, sin hablar directamente sobre la muerte. La subrogación está destinada a la familia, que no solo decide al final de la vida, sino que su papel cubre todo el proceso de la enfermedad, incluyendo el médico al que se asiste, las citas, en la medida que el tiempo y acompañamiento de estas dependen del tiempo de los cuidadores y familiares, por lo general las mujeres de la familia.

Tabla 1 Pacientes entrevistados y características sociodemográficas y de tratamiento.

\begin{tabular}{|l|l|l|l|l|l|l|}
\hline Paciente & Género & Edad & Religión & Tratamiento & Estado civil & Escolaridad \\
\hline Entrevista \#1 TR & F & 67 & Católica & $\begin{array}{l}\text { Terapia de reemplazo } \\
\text { renal }\end{array}$ & Divorciada & Básica Primaria \\
\hline Entrevista \#2 TR & M & 61 & Católica & $\begin{array}{l}\text { Terapia de reemplazo } \\
\text { renal }\end{array}$ & Casado & Postgrado \\
\hline Entrevista \#3 TR & F & 68 & NR & $\begin{array}{l}\text { Terapia de reemplazo } \\
\text { renal }\end{array}$ & Divorciada & Bachillerato completo \\
\hline Entrevista \#4 DP & $\mathrm{M}$ & 73 & Católica & Diálisis & Casado & Universitario \\
\hline Entrevista \#5 DP & $\mathrm{M}$ & 77 & Católica & Diálisis & Casado & Bachillerato \\
\hline Entrevista \#6 DP & $\mathrm{M}$ & 67 & Católico & Diálisis & Casado & Primaria completa \\
\hline Entrevista \#7 PD & $\mathrm{F}$ & 74 & Católica & Prediálisis & Separada & Primaria Completa \\
\hline Entrevista \#8 PD & $\mathrm{M}$ & 81 & Católica & Prediálisis & Casado & Primaria completa \\
\hline Entrevista \#9 PD & $\mathrm{M}$ & 80 & Católica & Prediálisis & Viudo & Tecnológico \\
\hline Entrevista \#10 HEMO & $\mathrm{M}$ & 75 & $\begin{array}{l}\text { Adventista del } \\
70 \text { día }\end{array}$ & Hemodiálisis & Casado & Primaria completa \\
\hline Entrevista \#11 HEMO & $\mathrm{M}$ & 81 & Católica & Hemodiálisis & Casado & Ninguna \\
\hline Entrevista \#12 HEMO & $\mathrm{M}$ & 72 & Católica & Hemodiálisis & Casado & Primaria completa \\
\hline
\end{tabular}


Para el caso de los pacientes aquí entrevistados, un paciente informa acerca del silencio completo de sus hijos ante la opción de trasplante renal, siendo ellos los posibles donantes. Durante las entrevistas no se evidenciaron actitudes dominantes de los familiares, excepto una constante necesidad de servir de repositorio de memoria para los pacientes, recordando medicamentos, si habían o no recibido información sobre sus tratamientos o sobre el pronóstico.

Los entrevistados presentan a sus familias como colaboradoras y acompañantes en el proceso, estando esto acorde con su situación y adherencia al tratamiento. La mayoría expresó su delegación de toma de decisiones a sus familiares, ante el evento de no poder decidir por sí mismos; pero se dejan concertados algunos aspectos acerca del funeral y manejo de los restos, que pueden incluir cremación, uso o no de cementerios, y rituales religiosos. Sin embargo, las decisiones médicas o relacionadas con el contexto clínico no son discutidas en vida, solo delegadas.

\section{El género}

Si bien se entrevistó a pocas mujeres, y se deberá profundizar en ello en el futuro, las contactadas manifestaron una necesidad de acompañamiento para sacar citas, recordar los medicamentos, ir a controles, hacer procedimientos burocráticos, decidir el tipo de tratamiento, su lugar habitual de vivienda o la necesidad de traslado, entre muchas otras decisiones que deben asumir en la vida cotidiana. Esto se percibe y expresa como "apoyo familiar" más que como dependencia. En las mujeres entrevistadas involucró a qué médico asistir y cuándo asistir, mediado por lo general por las hijas cuidadoras. $\mathrm{Al}$ no tener en el grupo de entrevistadas mujeres casadas, no es posible establecer relación entre la toma de decisiones y el cónyugue. Entre las pacientes entrevistadas, una mujer expresa haber sido abandonada por su pareja luego del diagnóstico: "Ese viejo me botó cuando me enfermé, no quiero ni que me pregunte, ese señor cada, porque me enfermé de tres partes, me botó y se fue con otra vieja” ( Entrevista TR \# 1).

En el caso de las mujeres entrevistadas, las tres divorciadas, sustentan su cuidado y decisiones en las relaciones con sus respectivas hijas.
Por otro lado, los hombres entrevistados se expresan más como individuos que toman decisiones por sí mismos. Al preguntar a un paciente sobre sus preocupaciones, responde: "P: Así como estoy pasando los días generalmente no [se preocupa], yo manejo todas mis finanzas, estoy en compañía, tengo un medio ambiente propicio, en el que he estado siempre, salgo a donde quiero, puedo ir solo, también me acompañan, pero más que todo solo" (Entrevista PD \# 9).

\section{La religión}

En las pacientes católicas entrevistadas, las decisiones de final de vida están mediadas por la relación con Dios, como ente que las expone y decide sobre sus vidas: "Toca aceptar lo que venga porque qué va a hacer uno, yo no voy a hacer ninguna cosa mala, nada de eso ni que me maten tampoco, ni me pongan nada de eso, me dejen morir como Dios me trajo a este mundo, Dios me dio la vida, Dios me la quita" (Entrevista TR \# 1). En este caso en particular, la autonomía se centra en seguir el dictamen de su fe, que en los imaginarios vincula decisiones de fin de vida con eutanasia o suicidio asistido. Las decisiones en este contexto deben considerarse de carácter heterónomo: A la pregunta de: “¿Usted les cedería la decisión a sus hijos?", la mujer responde: "No, no, yo... yo... le exigiría lo que le estoy diciendo a su merced: seguir el dictamen de Dios y, si ya ellos dicen... es cosa aparte..." (Entrevista TR \# 1).

Otra mujer, que no se identifica con ninguna religión, excepto con "ser buena persona", expresa lo siguiente cuando se le pregunta sobre las decisiones anticipadas:

"E: ¿El documento que firmó su hija, otras personas en trasplante renal también lo conocen?

P: Si, mis amigas conocen.

E: ¿Pero lo han firmado?

P: No, es eso la religión, la religión no les permite donar su cuerpo, no les permite hacer caridad con esto, aunque la verdad no hablamos de esto porque dejaríamos de ser amigas o, por lo menos, habría un espacio como ahí pues yo respeto mucho" (Entrevista TR \# 3). 
En los pacientes hombres son comunes las expresiones "Cuando mi Dios se acuerde de uno", refiriéndose al momento de la muerte, y a dejar seguir el "curso natural" de la enfermedad o la muerte. El tratamiento médico no se considera una intervención fuera de lo "natural", y la decisión sobre los tratamientos se deja a consideración de una entidad superior.

\section{Las mayores barreras para una autonomía so- lidaria: el tabú de la muerte y la comunicación con el personal de salud}

Dos tendencias surgen en las entrevistas: la muerte como lo indecible, en la medida que, si se piensa, se menciona o se habla de ella, se le atrae; y la muerte como algo lejano, una realidad que no les compete. Un paciente masculino, al preguntarle al respecto, responde: "(...) pues yo no quiero que me hablen de la muerte" (Entrevista DP \# 4). Si bien se habla de la muerte, se refieren en especial al manejo del cuerpo y funeral, y cómo quieren que se manejen sus restos, más que a decisiones que haya que tomar en el proceso de muerte. Otro paciente resalta: "(...) como uno no le para bolas a eso, nunca piensa en morirse, ¡qué va a pensar en morirse!" (Entrevista \# 2 TR). Finalmente, un familiar responde por el paciente: "E: ¿Habla de la muerte, del futuro con su familia? P: No, F: No le gusta a él no le gusta la muerte, ni que se la nombren" (Entrevista DP \# 6).

Finalmente, las decisiones que acompañan la ERCA tienen como actores centrales a los médicos tratantes y su relación con los pacientes. Estas están mediadas por el reconocimiento del saber médico como aquello que los mantiene vivos. Un paciente afirma: "yo no sé mucho de medicina, pues lo que me digan los médicos, yo me atengo a ellos me dicen haga esto o no lo haga, y yo hago lo mejor que me pueda" (Entrevista DP \# 5).

También, los pacientes señalan al personal de salud previo como fuente de desinformación, falta de comunicación efectiva e incluso de procedimientos no realizados o mal realizados, que hacen que el paciente pierda la confianza en el personal de salud. Destaca en estas entrevistas que los pacientes se refieren a sus médicos tratantes actuales, de la Unidad de Cuidado Renal y del Hospital San Ignacio, como cálidos, amables, que informan y dan opciones, comparándolos con otras instituciones. Ningún paciente se refiere a la calidad de la relación del médico con sus cuidadores o familiares; sin embargo, los familiares sí son referente y repositorio de la memoria de sus pacientes, sus medicamentos, procedimientos y demás requerimientos en el tratamiento o burocráticos, y esto se ve reflejado constantemente en las entrevistas.

Resalta el desconocimiento reportado sobre el DMD y las decisiones anticipadas, en los que la mayoría de los pacientes reportan que "nunca" se les hablado del tema. Solo los pacientes con mayor nivel de escolaridad hablan del asunto. Si consideramos la baja escolaridad de los pacientes, esta sensación de ausencia de información puede relacionarse más con una falta de comprensión, y por ende de reflexión, del proceso a seguir y del pronóstico de la enfermedad, necesarios para la toma de decisiones autónomas y solidarias.

\section{Discusión}

Los estudios realizados sobre autonomía y toma de decisiones se centran en su mayoría en la comunicación médico-paciente y rara vez incluyen otros actores en el proceso. En una revisión del tema(9) se identificó la importancia y necesidad de incluir a los integrantes de la familia en la toma de decisiones, en la medida en que pueden tener una influencia positiva o negativa en este proceso. La influencia positiva refuerza la relación con los pacientes y reduce la ansiedad en la toma de decisiones. Se considera negativa cuando los familiares interfieren con el proceso con actitudes dominantes, lo que genera tensión. También pueden tener una actitud pasiva, es decir que no estén involucrados o no tengan deseos de participar por razones específicas; o una actitud activa, en la que hacen parte del proceso.

Un estudio de Cervantes, en Denver, mostró cómo los participantes latinos prefieren la toma de decisiones en grupo familiar. Quienes no habían discutido previamente con la familia, expresaron preferir ceder la toma de decisiones a sus familias y confiar en que ellos tendrían en cuenta sus mejores intereses(26).

En una revisión reciente, se concluyó que la planificación del cuidado centrado en la familia, que 
implica el conocimiento de las voluntades anticipadas, lleva a una mejor comprensión de las preferencias de los pacientes para la atención al final de la vida, lo que conduce a un menor uso de tratamientos fútiles que prolongan la vida y a recurrir con mayor frecuencia a los servicios de atención paliativa y cuidados paliativos(27).

Los estudios basados en la autonomía solidaria señalan que la autonomía se alimenta del diálogo con los más cercanos, por lo que la toma de decisiones se podría considerar compartida, al involucrar no solamente al sujeto sobre el cual se toma la acción sino aquellas personas cuyas preocupaciones jugarían un papel crucial(28).

Considerar las interacciones dinámicas entre los miembros de la familia para la toma de decisiones, es esencial para la comprensión y el tratamiento de los trastornos individuales y relacionales. Hay evidencia sustancial de la influencia mutua del funcionamiento familiar, la salud y las enfermedades, y la utilidad de intervenciones centradas en la familia en pacientes con afecciones de salud crónicas frente a la toma de decisiones conjuntas(29).

En el adulto mayor es necesario considerar su dependencia funcional $y$, en particular, la relacionada con la percepción de apoyo y el género. Estudios previos muestran que, al analizar la percepción de apoyo familiar por género, esta fue mejor para las mujeres que para los hombres, y las mujeres mostraron menor dependencia funcional. Sin embargo, esta independencia funcional no necesariamente está directamente relacionada con el desarrollo de la autonomía individual, en la medida en que las acciones, roles, labores e imaginarios sitúan a la mujer como cuidadora y no como sujeto de cuidado(30).

El género, comprendido en el marco del modelo de determinantes sociales de la salud(31), debe comprenderse vinculado a otros determinantes, como empleo, educación, lugar de vivienda entre otros. En particular para las mujeres más pobres, rurales, de bajo nivel educativo, se encontrarán mayores inequidades en la medida en que ellas y sus decisiones están supeditadas a la aprobación o consentimiento de los hombres - esposo o hijos- con quienes habite(31), pero también con formas particulares de la trayectoria de vida de la mujer y su desarrollo psicosocial(32), así como con su grado de dependencia funcional.

Adicionalmente, el cuidado de la persona mayor recae por lo general en las mujeres de la siguiente generación, mientras que el cuidado de los hombres heterosexuales mayores recae en su cónyuge mujer.

En este análisis se propone como hipótesis, para futuras investigaciones, que aquellas mujeres en las que persistan relaciones de poder, dependencia emocional o económica, y que tengan un lugar menor en la jerarquía o estructura familiar, delegarán su autonomía al resto de la familia, en especial a sus hijas, al ser una conducta persistente o heredada de un rol de sumisión.

La forma de relacionamiento y de estructura familiar varía de sociedad a sociedad y, dentro de esta, de acuerdo con el género, ingresos económicos, educación, etnia y, en especial, cultura, en la que el tabú de la muerte y el miedo a hablar sobre la muerte aparece como transversal a los participantes(33), asociado a su religión. Se supone que este miedo atraviesa los problemas de comunicación entre pacientes, familiares y médicos, y que se requiere un ejercicio por parte del médico tratante para abordar la muerte y la posibilidad de la muerte como un campo en el que el papel educador del médico es requerido.

\section{Conclusiones}

Es importante generar procesos de autorreflexión y autonomía solidaria, en los que la determinación surja del minucioso autoanálisis de su realidad y la respectiva consideración empática hacia las personas y familiares, quienes potencialmente deberán asumir la toma de decisiones de sus familiares, con los consiguientes efectos traumáticos que derivarían de dicha situación. Este actuar no es accesorio: forma parte de la verdadera finalidad de la práctica médica, como es cuidar integralmente al paciente más que curar. Por eso, educar al paciente, entablar relación con la familia y acompańarla en el proceso de toma de decisiones enfatiza en el carácter pedagógico de la medicina. El personal de salud estaría llamado a generar espacios de reflexión al respecto, estimulando el juicio moral autónomo en los pacientes que aún tienen sus fa- 
cultades en equilibrio, para que las voluntades anticipadas sean el resultado de un juicioso ejercicio relacional, consecuencia de la interacción consigo mismo y con su familia, reconociendo las diversas estructuras y dinámicas familiares posibles.

Podría afirmarse que, para el grupo de pacientes entrevistados, la educación, el género y la dependencia económica están relacionados estructuralmente con la autonomía, y que, en los casos de pacientes de origen rural, baja escolaridad, mujeres y personas mayores, la subrogación de la decisión es lo que más ocurre, siendo coherente con estudios previos.

En todas las entrevistas surgió la familia como actor importante en la difusión y apropiación de la información, por lo que se debe trabajar de manera exhaustiva en las estrategias de una mejor comunicación médico-familia y hacer retroalimentación para obtener mejores resultados, incorporando a todos los actores, tanto familiares como al personal de apoyo de la unidad renal.

Se identificó, de manera preliminar, la relación entre género, educación, ingreso/dependencia económica y dependencia funcional como determinantes importantes a ser explorados en el cuidado, DMD y decisiones anticipadas en la ERCA, sin investigaciones previas identificadas en nuestro contexto.

Si durante la segunda mitad del siglo XX se empezó a considerar que el fin de la medicina no era propiamente "curar enfermedades" sino cuidar de la persona, como categoría que abarca la anterior y la supera, en el siglo XXI corresponderá reforzar la idea del cuidado de la persona como resultado de complejos procesos sociales y, más aún, de procesos que involucren el fin de la vida.

\section{Referencias}

1. Alonso Babarro A, García Llana H, Leiva Santos JP, Sánchez Hernández R. Cuidados Paliativos en enfermedad renal crónica avanzada. Sociedad Española de Nefrología S.E.N. Sociedad Española de Cuidados Paliativos SECPAL2018. Disponible en: https://www.revistanefrologia.com/es-cuidados-paliativos-enfermedad-renal-cronica-articulo-X1888970018627957

2. Lopera-Medina MM. La enfermedad renal crónica en Colombia: necesidades en salud y respuesta del Sistema General de Seguridad Social en Salud. Revista Gerencia y Políticas de salud 2016; 15: 212-33. Disponible en: http://10.11144/ Javeriana.rgyps15-30.ercc

3. Mercado-Martínez FJ, Hernández-Ibarra E. Chronic illness from the perspective of patients and health professionals: a qualitative study in Mexico. Cad Saúde Pública 2007; 239: 2178-86. Disponible en: https://.org/10.1590/S0102311X2007000900025

4. Luis Franco P. Reflexiones acerca del fin de vida en enfermedad renal crónica Estadio 5. Universidad Pública de Navarra Nafarroako Unibertsitate Publikoa; 2017. Disponible en: https://hdl.handle.net/2454/23439

5. Muscat DM, Kanagaratnam R, Shepherd HL, Sud K, McCaffery K, Webster A. Beyond dialysis decisions: a qualitative exploration of decision-making among culturally and linguistically diverse adults with chronic kidney disease on haemodialysis. BMC Nephrol. 2018; 19. Disponible en: http://10.1186/s12882-018-1131-y

6. Espíndola Lira JA. Regresión Psicomotriz. 2019. En: Práctica de Geriatría [Internet]. McGraw-Hill Medical. Disponible en: https://accessmedicina. $\mathrm{mhmedical} . \mathrm{com} /$ content. aspx?bookid=1500\&sectionid=98098258

7. Heras Benito M, Fernández-Reyes Luis MJ. Toma de decisiones compartida en la enfermedad renal crónica avanzada del anciano. Shared decision-making in advanced chronic kidney disease in the elderly English 2019; 1525: 188-94. Disponible en: https://10.1016/j.medcli.2018.07.011

8. Puyol Á. Hay bioética más allá de la autonomía. Revista de Bioética y Derecho 2012; 45-58. Disponible en: https://. org/10.1344/rbd2012.25.7538

9. Lamore K, Montalescot L, Untas A. Treatment decision-making in chronic diseases: What are the family members' roles, needs and attitudes? A systematic review. PubMed - NCBI. Patient education and counseling 2017; 10012 :2172-81. Disponible en: http://10.1016/j.pec.2017.08.003

10. Pérez MGR. Sujeto y corporalidad: la experiencia del padecimiento en contextos de hospitalización; 2011. Disponible en: http://repositorio.ciesas.edu.mx//handle/123456789/42

11. Lobato A. Representaciones sociales de los docentes sobre la investigación de las facultades de educación. Antecedentes, tendencias y ausencias. Revista de Educación y desarrollo social 2010; 2: 130-42. Disponible en: https://.org/10.18359/ reds.919 
Toma de decisiones centradas en la familia en la enfermedad renal crónica avanzada - Laura Margarita Forero et al.

12. Oberti P. El estudio de las representaciones sociales como aporte para las intervenciones profesionales. Fronteras 2015; 8: 157-65. Disponible en: http://hdl.handle.net/20.500.12008/7254

13. Clinical Practice Guideline for the Evaluation and Management of Chronic Kidney Disease CKD. Kidney International Suplements 2013; 31. Disponible en: https://kdigo.org/wp-content/uploads/2017/02/KDIGO_2012_CKD_GL.pdf

14. Beca I, JP. Derecho a morir: un debate actual. Rev Médica de Chile 2005; 1335: 601-6. Disponible en http://dx.. org/10.4067/S0034-98872005000500014

15. Ministerio de Salud. República de Colombia. Resolución 2665. Derecho a suscribir el Documento de Voluntad Anticipada, junio 2018. Disponible en: https://www.minsalud.gov.co/Normatividad_Nuevo/Resoluci\%C3\%B3n\%20No.\%20 2665\%20de\%202018.pdf

16. Piperberg. M. La persona y el debate bioético sobre el final de la vida. Universitat de Barcelona; 2015. Disponible en: http:// hdl.handle.net/2445/98700

17. Kipper DJ. Límites del poder familiar en las decisiones acerca de la salud de sus hijos-directrices. Revista Bioética 2015; 231: 40-50. Disponible en: https://.org/10.1590/1983-80422015231044

18. Beauchamp T, Childress J. Principies of Biomedical Ethics. Nueva York: Oxford University Press; 2001.

19. Iosa FJ. Autonomía personal y reflexión: un análisis de las ideas de Harry Frankfurt y Gerald Dworkin. Revista Eletrônica do Curso de Direito da UFSM 2017; 121: 272-97. Disponible en: http://dx..org/10.5902/1981369426370

20. Ramírez S, Gabriela. M. Autonomía moral: Una posibilidad para el desarrollo humano desde la ética de la responsabilidad solidaria. Revista de Psicología 2003; 121: 35. Disponible en: http://www.redalyc.org/articulo.oa?id=26400102

21. Mackenzie C. Concepciones de la autonomía y concepciones del cuerpo en la bioética. En: Rivera ML, Editor. Feminist Bioethics At the Center, On the Margins. Bogotá; 2010: 71-90.

22. Elwyn G, Frosch D, Thomson R, Joseph-Williams N, Lloyd A, Kinnersley P, et al. Shared decision making: a model for clinical practice. Journal of general internal medicine 2012; 2710: 1361-7. Disponible en: https://link.springer.com/ article/10.1007/s11606-012-2077-6

23. García GE. Neuropsicología del comportamiento moral. Neuronas Espejo, funciones ejecutivas y ética universal. En: Torre Jdl, Editor. Neurociencia, Neuroética y Bioética. Madrid: Universidad Pontificia Comillas; 2014: 43-75.

24. Angrino Quebradas DA. El error de Descartes: La emoción, la razón y el cerebro Humano. Cuadernos de neuropsicología 2011; 52: 173-8. Disponible en: http://pepsic.bvsalud.org/scielo.php?script=sci_arttext\&pid=S0718$41232011000200006 \& \operatorname{lng}=\mathrm{pt} \& \mathrm{nrm}=$ iso

25. Camps V. El gobierno de las emociones. Herder Editorial; 2011.

26. Cervantes L, Jones J, Linas S, Fischer S. Qualitative Interviews Exploring Palliative Care Perspectives of Latinos on Dialysis. Clin J Am Soc Nephrol. 2017; 125: 788-98. Disponible en: https://.org/10.2215/CJN.10260916

27. O'Halloran P, Noble, H, Norwood, K, Maxwell P, Shields J. Advance care planning with patients who have end-stage kidney disease: a systematic realist review. Journal of Pain and Symptom Management 2018; 56(5): 795-807. Disponible en: https://.org/10.1016/j.jpainsymman.2018.07.008

28. Taylor JS, The College of New Jersey E, New Jersey, USA. Social Autonomy and Family-Based Informed Consent. The Journal of Medicine and Philosophy: A Forum for Bioethics and Philosophy of Medicine 2019; 445: 621-39. Disponible en: https://.org/10.1093/jmp/jhz020

29. Rolland J, Emanuel L, Torke A. Applying a family systems lens to proxy decision making in clinical practice and research. Families, Systems, \& Health 2017; 35: 7. Disponible en: https://10.1037/fsh0000250

30. Jiménez-Aguilera B, Baillet-Esquivel LE, Ávalos-Pérez F, Campos-Aragón L. Dependencia funcional y percepción de apoyo familiar en el adulto mayor. Atención Familiar 2016; 23(4): 129-33. Disponible en: https://.org/10.1016/j. af.2016.08.002

31. Organización Mundial de la Salud. Determinantes sociales de la salud. Organización Mundial de la Salud; 2012. Disponible en http://www.who.int/social_determinants/es/. [consultada el 13 de marzo de 2020].

32. Yuni JA, Urbano CA. Envejecimiento y género: perspectivas teóricas y aproximaciones al envejecimiento femenino. Revista Argentina de Sociología [Internet] 2008; 610: 151-169. Disponible en: https://www.redalyc.org/articulo.oa?id=26961011

33. Herrero PR, Gascón AdlH, Selva MC. Antecedentes de la pedagogía de la muerte en España. Enseñanza \& Teaching 2012; 30(2): 175-95. Disponible en: https://revistas.usal.es/index.php/0212-5374/article/view/9320/9613

Recibido: 8 de junio de 2020

Aceptado: 2 de julio de 2020 